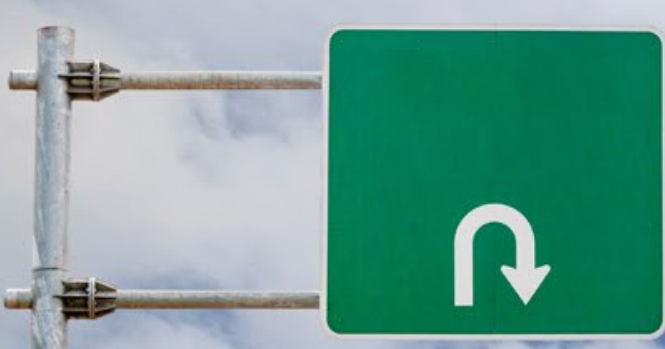

\title{
Kehrtwende der Großen Koalition in der PEPP-Diskussion
}

Letztes Jahr endete das Editorial in der März-Ausgabe 2015 zur drohenden Einführung eines pauschalierten Entgeltsystems in Psychiatrie und Psychosomatik (PEPP) mit dem Satz: „So ist erfreulich, dass sich ein breiter medizinisch begründeter Widerstand gegen eine vor allen Dingen bürokratisch und finanztechnisch begründete Neugestaltung der Finanzierung psychiatrisch-psychosomatisch-psychotherapeutischer Krankenhausleistungen formiert." Am 18. Februar 2016 hat die Große Koalition diesen Bedenken Rechnung getragen und eine massive Kursänderung vollzogen. Mit ausschlaggebend dafür dürfte ein im September 2015 dem Gesundheitsministerium übergebenes Alternativkonzept der DGPPN sowie 19 weiterer Fachgesellschaften und Verbände gewesen sein. In einem ersten Schritt beschloss am 8. Januar 2016 die SPD-Bundestagsfraktion, dass das von der schwarz-gelben Koalition unter Gesundheitsminister Daniel Bahr entwickelte PEPP-System nicht weiterverfolgt werden sollte. Dem schloss sich Bundesgesundheitsminister Hermann Gröhe an, so dass ein wesentlich verbessertes Eckpunktepapier in der Großen Koalition konsentiert werden konnte. Es beinhaltet die folgenden Punkte: Es wird zwar an den Grundsätzen von Leistungsorientierung und mehr Transparenz festgehalten, jedoch wird in einer Neuausrichtung des PsychEntgeltsystems Verhandlungspartnern vor Ort die Möglichkeit gegeben, unter Berücksichtigung regionaler Bedingungen und hausindividueller Besonderheiten bedarfs- und leistungsgerechte Budgets zu vereinbaren. Damit entfällt die im PEPP-System vorgesehene Landeskonvergenz. Das heißt, auf der Grundlage eines bundesweiten und empirisch kalkulierten Entgeltkatalogs wird das Budget der einzelnen Einrichtungen unter Berücksichtigung von leistungsbezogenen strukturellen Besonderheiten (z.B. regionale Versorgungsverpflichtung) vereinbart.

Bundeseinheitliche Bewertungsrelationen sollen aufgrund der Kostendaten von repräsentativen Kalkulationshäusern ermittelt werden. Darüber hinaus wird der Gemeinsame Bundesausschuss beauftragt, verbindliche, auf Leitlinien gestützte Mindestvorgaben zur Personalausstattung zu erarbeiten. Diese Mindestanforderungen sollten zunächst bei Indikationen definiert werden, für die bereits jetzt evidenzbasierte S3-Leitlinien vorliegen. Im Gegensatz zum initialen PEPP-System soll also jetzt normativ der Personalbedarf für eine gute Versorgungsqualität entsprechend den wissenschaftlichen Standards ermittelt werden. In der Übergangsphase soll für die Kalkulationshäuser eine 100 \%ige Umsetzung der Psychiatriepersonalverordnung (PsychPV) vorgegeben werden. Die Selbstverwaltungspartner sollen

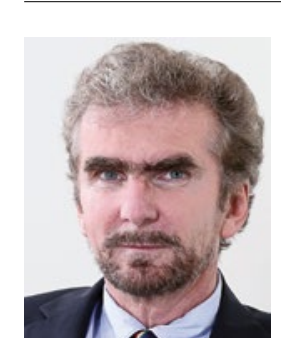

Prof. Dr. med. Mathias Berger

Ärztlicher Direktor der Klinik

für Psychiatrie und Psychotherapie

Universitätsklinikum Freiburg

E-Mail: mathias.berger@uniklinik-freiburg.de

auf Bundesebene einen Krankenhausvergleich entwickeln, der als Transparenzinstrument auf lokaler Ebene den Vertragspartnern ermöglichen soll, ein auf das jeweilige Krankenhaus bezogenes individuelles Budget zu vereinbaren.

Ein weiterer entscheidender Fortschritt des Eckpunktepapiers ist ein erster Schritt zur Stärkung der sektorenübergreifenden Versorgung durch Einführung einer komplexen psychiatrisch-psychotherapeutischen Akutbehandlung im häuslichen Umfeld (Hometreatment). Dies soll die Flexibilität und Bedarfsgerechtigkeit der Versorgung erhöhen und wird aus der Krankenhausvergütung erstattet. Die Neuausrichtung der gesetzlichen Rahmenbedingungen für das neue Psych-Entgeltsystem wird 2016 erfolgen und soll ab 2017 verbindlich von allen Psych-Einrichtungen unter budgetneutralen Bedingungen angewendet werden.

\section{Fazit}

Bei dem Eckpunktepapier handelt es sich entsprechend der Einführung der PsychPV Anfang der 1990er-Jahre um eine politische Entscheidung: Die Behandlung psychisch Erkrankter soll sich im stationären und teilstationären Bereich - wie in der gesamten übrigen Medizin - am wissenschaftlichen Fortschritt orientieren. Die offensichtlichen Schwächen der ambulanten Versorgung sollen, zumindest bei sehr schwer kranken Patienten, nicht nur durch die psychiatrischen Institutsambulanzen, sondern auch durch das Hometreatment zumindest teilweise kompensiert werden.

Überrascht und erfreut ist zur Kenntnis zu nehmen, dass intensive Diskussionsprozesse mit der Politik erfolgreich durchgeführt werden können und einschneidende Korrekturen auch in einem bereits fortgeschrittenen gesetzlichen Entwicklungsprozess möglich sind. 